TITLE:

\title{
Dispersion relations of director fluctuations along the direction perpendicular to the helical axis in cholesteric liquid crystals
}

\section{AUTHOR(S):}

Yoshioka, J.; Takanishi, Y.; Yamamoto, J.

\section{CITATION:}

Yoshioka, J.... [et al]. Dispersion relations of director fluctuations along the direction perpendicular to the helical axis in cholesteric liquid crystals. EPL (Europhysics Letters) 2012, 98(1): 16006.

ISSUE DATE:

2012-04-01

URL:

http://hdl.handle.net/2433/155560

\section{RIGHT:}

(c) IOP Publishing 2012; This is not the published version. Please cite only the published version.; この論文は出版社版でありません。引用の 際には出版社版をご確認ご利用ください。 
Dispersion relations of director fluctuations along the direction perpendicular to the helical axis in cholesteric liquid crystals

J. Yoshioka, Y. Takanishi and J. Yamamoto

Department of Physics, Graduate School of Science, Kyoto University

Kita-Shirakawa, Kyoto 606-8502, Japan

PACs number: 61.30.-v, 42.70.Df, 78.35.+c

We theoretically analyzed dispersion relations of director fluctuations along the direction perpendicular to the helical axis in cholesteric liquid crystals, and experimentally verified validity of the analysis by dynamic light scattering (DLS) measurement. It is found that the dispersion relations are well-explained by two independent modes, which we call 'splay-bend' and 'undulation' modes. 
After invention of the polymer stabilized blue phase in 2002, development of the next-generation displays utilizing fast response of Kerr effect in the blue phase has been extensively performed [1-3].

3 However, its dynamic response against the electric field is not so sufficiently understood because of complexity caused by the existence of the three-dimensional lattice composed of double twist cylinders, in which cholesteric helical axes are locally extended radially in the plane normal to the cylinder rod. In fact, the hydrodynamic modes of the orientation fluctuations are not described completely even in the conventional cholesteric phase. Pseudo layer structure exists along the helix in the cholesteric phase, and can be responded elastically against the uniaxial dilation or compression of the helix. Thus, eigen modes for the distortion of director in the nematic phase are forbidden or coupled with other modes as similar to the smectic phase. In order to understand the relation between dynamics and response of the director in the blue phase, it is important to understand the description of complete set of the hydrodynamic modes in the cholesteric phase first.

In the nematic phase, hydrodynamic modes of the orientation fluctuation have been completely described by the individual three modes called splay, twist and bend modes [4]. On the other hand, in the cholesteric phase, the hydrodynamic modes depend on whether wave vector is along parallel or perpendicular to its helical axis. The mode parallel to the helical axis has already been analyzed theoretically, and it has turned out that the dispersion relations are described by two modes, which are called 'twist' and 'viscous-splay' ('umbrella') modes [4-6]. They have also been verified experimentally, using dynamic light scattering (DLS) [7, 8]. Later, fluctuations of tensor order-parameter modes parallel to the helical axis are also calculated [9]. In contrast, for the mode perpendicular to the helical axis, there are few investigations, which have only been done for the case in which the wave number of scattering vector $q$, which is defined as the difference between the wave number vectors of the incident and the scattered light is much less than that of the helical pitch $q_{0}$, which is defined by $q_{0}=2 \pi / P$, where $P$ is helical pitch length $\left(q<<q_{0}\right)[6,10]$. By the way, order-parameter fluctuations in the isotropic phase above the cholesteric phase are also investigated 
as the pretransitional behavior $[11,12]$.

In this paper, we focus on the hydrodynamic modes perpendicular to the helical axis in the

3 cholesteric phase. Without assuming $q<<q_{0}$, we analytically calculated the dispersion relations of

4 the fluctuations in case that the scattering vector is perpendicular to the helical axis, and verified

$5 \quad$ validity of the analysis experimentally using DLS.

6 From the macroscopic point of view, it can be considered that the cholesteric liquid crystal has

7 pseudo-layer structure derived from its helix, and we assumed that it deforms without breaking and compressing the pseudo-layer structure. According to the layer deformation in the smectic A phase, small deformation of the pseudo-layer structure is written by the displacement $u$ from the equilibrium configuration as shown in Fig. 1(b), and the increase of the elastic free energy is written using $u$ as

11 similar to the smectic phase. On the other hand, the elastic free energy density per unit volume of

12 the cholesteric liquid crystal is usually written using the director $\mathbf{n}$ [4]. Small deformation of the director from the equilibrium configuration $\boldsymbol{\delta} \mathbf{n}$ has two components $\delta n_{\phi}$ and $\delta n_{z}$ along perpendicular and parallel to the helical axis, where we assumed that $\mathrm{z}$ axis is parallel to the helical axis in the non-deformed state. Using them, we can write the director with small deformation as follows,

$$
\mathbf{n}=\left(\cos q_{0} z-\delta n_{\phi} \sin q_{0} z, \sin q_{0} z+\delta n_{\phi} \cos q_{0} z, \delta n_{z}\right)
$$

Although $\delta n_{\phi}$ is independent of $u$, there is a relationship between $\delta n_{z}$ and $u$,

$$
\delta n_{z}=\frac{\partial u(r, \phi)}{\partial r} \cos \left(\phi-q_{0} z\right)
$$

where we adopted cylindrical coordinate system $(r, \phi, z)$. Hence, we calculated total elastic free energy $F$ derived from the small fluctuations, using $\delta n_{\phi}$, and $u$ instead of $\delta n_{z}$. We expand $F$ in a

21 Fourier series,

$$
F=\frac{1}{2 V} \sum_{\mathbf{k}}\left[\left.\left(\frac{K_{1}+K_{3}}{2} k_{r}^{2}+K_{2} k_{z}^{2}\right) \delta n_{\phi}(\mathbf{k})\right|^{2}+\left.\frac{3 K_{3}}{8} k_{r}^{4}|u(\mathbf{k})|^{2}\right|_{\rfloor},\right.
$$

where $K_{1}, K_{2}$ and $K_{3}$ are elastic constants corresponding to splay, twist, and bend deformations of 
1 local nematic director respectively, and $V$ is volume of a sample. $\quad k_{r}$ and $k_{z}$ are wavenumbers nomal

2 and parallel to the helical axis, respectively.

3 Using the total free energy expressed in eq. (3), we calculated hydrodynamic equations for $\delta n_{\phi}$ and

$4 u$ respectively. Here, we assume that the flow field is zero. From the balance of molecular field

5 and rotational viscosity, the hydrodynamic equation for $\delta n_{\phi}$ is written as

$6 \quad-\left(\frac{K_{1}+K_{3}}{2} k_{r}^{2}+K_{2} k_{z}^{2}\right) \delta n_{\phi}(\mathbf{k})=\gamma_{1} \frac{d \delta n_{\phi}(\mathbf{k})}{d t}$,

where $\gamma_{1}$ is a rotational viscosity. Time evolution of $\delta n_{\phi}$ indicates the exponential decay function, and its relaxation frequency $1 / \tau_{\phi}$ is written as

$9 \quad \frac{1}{\tau_{\phi}}=\frac{\left(K_{1}+K_{3}\right)}{2 \gamma_{1}} k_{r}^{2}+\frac{K_{2}}{\gamma_{1}} k_{z}^{2}$.

10 We call this relaxation 'splay-bend mode', which shows splay and bend deformations of the

11 director in the plane perpendicular to the helical axis as shown in Fig. 1(a). From eq. (5), it is found

12 that this mode has both components parallel and perpendicular to the helical axis. This expression

13 is the same as the 'phase mode' which is one of the director fluctuation modes observed in the chiral

14 smectic $C^{*}$ phase with a helical structure $[13,14]$.

15 On the other hand, from the analogy with lyotropic lamellar phase, the hydrodynamic equation for

$16 u$ is written as [15]

$$
-\frac{3 K_{3}}{8} k_{r}^{2} u(\mathbf{k})=\eta \frac{d u(\mathbf{k})}{d t}
$$

18 where $\eta$ is a viscosity constant for the undulation deformation. Time evolution of $u$ also indicates

19 the exponential decay function, and its relaxation frequency $1 / \tau_{u}$ is written as

$20 \quad \frac{1}{\tau_{u}}=\frac{3 K_{3}}{8 \eta} k_{r}^{2}$.

21 We call this relaxation 'undulation mode', which shows the undulation deformation of the pseudo-layer structure as shown in Fig. 1(b). Eq. (7) indicates that this mode has only the 
component normal to the helical axis.

We measured experimentally the relaxation frequencies of the two modes in two scattering geometries as shown in Figs. 2(a) and (b), which are suitable for measuring the splay-bend and undulation modes, respectively. In Fig. 2(a), the helical axis is parallel to the incident light, while it is perpendicular to scattering plane in Fig. 2(b). Here, we measured them with the scattering angle $\theta$ being relatively small (18 45 degrees), so that the scattering vector $\mathbf{q}$ is almost perpendicular to the helical axis in both of the two geometries, and the director fluctuation modes having $k_{r}$ are observed in the two geometries. The scattering vector $\mathbf{q}$ is defined by $\mathbf{q}=\mathbf{k}_{\mathbf{i}}-\mathbf{k}_{\mathbf{f}}$, where $\mathbf{k}_{\mathbf{i}}$ and $\mathbf{k}_{\mathbf{f}}$ are wave number vectors of incident and scattered lights respectively, and wave number of scattering vector is written as $q=|\mathbf{q}|=4 \pi n / \lambda$ assuming quasi-elastic scattering, where $n$ is the mean refractive index of cholesteric liquid crystals and $\lambda$ wave length of incident light. It is noted that both splay-bend and undulation modes do not include the scattering geometry where the scattering vector is near parallel to the helical axis, as a consequence of the incompressible condition of the layer of the helix.

The scattered field derived from director fluctuations is proportional to $\mathbf{f} \cdot(\boldsymbol{\varepsilon}(\mathbf{q}, t) \mathbf{i})$, where $\boldsymbol{\varepsilon}(\mathbf{q}, t)$ is the Fourier transformation of the relative dielectric tensor $\varepsilon(\mathbf{r}, t)$, and $\mathbf{i}$ and $\mathbf{f}$ are polarizations of incident and scattered lights respectively [4]. The $\boldsymbol{\varepsilon}(\mathbf{r}, t)$ of the cholesteric liquid crystals is written as $\varepsilon_{\alpha \beta}=\varepsilon_{\perp} \delta_{\alpha \beta}+\varepsilon_{a} n_{\alpha} n_{\beta}$, where $\varepsilon_{\perp}$ is the dielectric constant for the ordinary light, and $\varepsilon_{a}$ is the dielectric anisotropy. Furthermore, we assumed that the polarizations of the incident and the scattered light are not modulated even in the cholesteric samples. Although this assumption is quite different from real system, it is much more difficult and complex to consider the polarization of incident and scattered light strictly in the cholesteric liquid crystals. In the previous studies as shown in references $[5,7,8]$, the autocorrelation functions were theoretically analyzed without discussing the modulations of the polarizations in the cholesteric samples, and experimental results were well-explained using the analysis, so that we adopted the above assumption. Using them, we calculated time autocorrelation functions of scattering intensity, which is proportional to $<\mathbf{f} \cdot(\boldsymbol{\varepsilon}(\mathbf{q}, 0) \mathbf{i}) \mathbf{f} \cdot\left(\boldsymbol{\varepsilon}^{*}(\mathbf{q}, t) \mathbf{i}\right)>^{2}$ in the two scattering geometries. As a result, it is found that the undulation modes are observed with wave numbers $\mathbf{k}=\mathbf{q}+\mathbf{q}_{\mathbf{0}}$ and $\mathbf{q}-\mathbf{q}_{\mathbf{0}}$ in Figs. 2(a) and (b), while the splay-bend mode is only observed with wave numbers $\mathbf{k}=\mathbf{q}+\mathbf{2} \mathbf{q}_{\mathbf{0}}$ and $\mathbf{q}-\mathbf{2} \mathbf{q}_{\mathbf{0}}$ in Fig. 2(a), where 
$1 \mathbf{q}_{0}$ is defined by $\mathbf{q}_{0}=\left(0,0, q_{0}\right)$. Hence we call Fig. 2(a) 'splay-bend geometry' and Fig. 2(b) 2 'undulation geometry'. Substituting these conditions into eqs. (5) and (7), the relaxation

3 frequencies of the two modes are given by

4

$5 \quad \frac{1}{\tau_{u}}=\frac{3 K_{3}}{8 \eta} q^{2}$.

The experiment was performed in the cholesteric phase of the mixture of 7CB, MLC6427 and MLC6248, which is optical isomer of MLC6247 (Merck). We fixed weight ratio of the 7CB and the chiral dopant mixture of MLC6247 and MLC6248 to be 7:3. Furthermore, adjusting weight ratio of MLC6247 and MLC6248, we prepared the samples whose physical pitch lengths are between 300 and $820 \mathrm{~nm}$. Not depending on the mixing ratio, the mixtures showed almost the same phase sequence, which is isotropic $-42^{2} \mathrm{C}-$ cholesteric. In this experiment, we fixed the temperature at $30^{\circ} \mathrm{C}$. For the DLS measurement, we prepared $10 \mu \mathrm{m}$ thick sandwich cells made of two glass substrates. Since the incident light is almost perpendicular to the substrates in our measurement system, we need to align the helical axis perpendicular to the substrates for the splay-bend geometry (Fig. 2(a)), parallel to them for the undulation geometry (Fig. 2(b)). Hence, for the splay-bend geometry, we used commercial homogeneous planar cells, while for the undulation geometry, we adopted the method as shown in reference [16] to achieve the alignments in which the helical axis is parallel to the substrates.

Figure 3 shows the normalized autocorrelation functions of scattering intensity $g_{2}(t)-1$ actually observed in the splay-bend (symbol $\circ$ ) and undulation (symbol $\square$ ) geometries respectively. In the splay-bend geometry, two relaxations are observed around $\sim 0.04 \mathrm{~ms}$ and $\sim 0.8 \mathrm{~ms}$, while in the undulation geometry, single relaxation is observed around $\sim 0.8 \mathrm{~ms}$, which is close to the slower mode observed in the splay-bend geometry. Hence, it is confirmed that the faster mode in the splay-bend geometry corresponds to the splay-bend mode, and the slower mode in the splay-bend and the single 
mode in the undulation geometry correspond to the undulation mode, as described in the above section.

Figure 4(a) shows the dispersion relations of the relaxation frequencies of the splay-bend mode

with respect to the wave numbers. The relaxation frequencies are proportional to the square of the wave numbers, and its slope is independent of the helical pitch, while they show finite values depending on the pitch in the limit $q \rightarrow 0$, which are proportional to the square of the wave numbers of helical pitches as shown in Fig. 5. These behaviors are well-explained by eq. (8a). Figure 4(b) shows the dispersion relations of the undulation mode measured in the undulation geometry. The relaxation frequencies are proportional to the square of the wave numbers, and they become zero in the limit $q \rightarrow 0$, not depending on the helical pitches. These behaviors are also well-explained by eq. $(8 b)$.

To summarize, we theoretically analyzed dispersion relations of director fluctuations along the direction perpendicular to the helical axis in the cholesteric liquid crystal without assumption of $q<<q_{0}$, and verified validity of the analysis using DLS measurement. In the analysis, assuming that the cholesteric liquid crystals fluctuate without breaking its incompressible pseudo-layer structures, we designed the 'splay-bend' and the 'undulation' modes as the director fluctuation modes, which are expressed by eq. (8a) and (8b). Furthermore, it is found that the experimental results well satisfies with our proposed dispersion relation of the two modes, and the assumption mentioned above is also adequate.

\section{Acknowledgement}

We acknowledge Merck Ltd., Japan for supplying chiral dopants, MLC6247 and MLC6248. This work was supported by the Grant-in-Aid for Scientific Research (B) (No.21740312), the JSPS 
1 Core-to-Core Program "International research network for non-equilibrium dynamics of soft

2 matter' and the Global COE Program "The Next Generation of Physics, Spun from Universality

3 and Emergence" from the Ministry of Education, Culture, Sports, Science and Technology

4 (MEXT) of Japan.

5

6

16 References

17 [1] KIKUCHI H., YOKOTA M., HISAKADO Y., YANG H. and KAJIYAMA T., Nature Materials, $181(2002) 64$.

19 [2] GE Z., GAUZA S., JIANO M., XIANYU H. and WU ST., Appl. Phys. Lett., 94 (2009) 101104

20 [3] RAO L., YAN J., WU ST., YAMAMOTO S. and HASEBA Y., Appl. Phys. Lett., 98 (2011) $21 \quad 081109$

22 [4] DE GENNES P. G. and PROST J., The Physics of Liquid Crystals second edition, (Clarendon 
1 Press Oxford) 1993.

2 [5] FAN C., KRAMER L. and STEPHEN M. J., Phys. Rev. A, 2 (1970) 2482.

3 [6] LUBENSKY T. C., Phys. Rev. A, 6 (1972) 452.

4 [7] BORSALI R., SCHROEDER U. P., YOON D. Y. and PECORA R., Phys. Rev. E, 58 (1998)

$5 \quad \mathrm{R} 2717$.

6 [8] GIRIDHAR M. S. and SURESH K. A., Eur. Phys. J. E, 7 (2002) 167.

7 [9] PANTEA M. A. and KEYES P. H., Phys. Rev. E, 71 (2005) 031707.

8 [10] ADORJAN A., STOJADINOVIC S., SUKHOMLINOVIA L., TWEIG R. and SPRUNT S.,

$9 \quad$ Phys. Rev. Lett., 90 (2003) 035503.

10 [11] YANG C. C., Phys. Rev. Lett. 28 (1972) 955.

11 [12] HARADA T., and CROOKER P. P., Phys. Rev. Lett., 34 (1975) 20.

12 [13] DREVENSEK I., MUSEVIC I. and COPIC M., Phys. Rev. A, 41 (1990) 923.

13 [14] SUN H., ORIHARA H. and ISHIBASHI Y., J. Phys. Soc. Jpn., 60 (1991) 4175.

14 [15] NALlET F., ROUX D. and PROST J., J. Phys. France, 50 (1989) 3147.

15

[16] HUNG W. -C., CHENG W. -H., LIN Y. -S., JANG D. -J., JIANG I. -M. and TSAI M. -S., J.

16 Appl. Phys., 104 (2008) 063196.

17

18

19

20 
20 Figure caption

21 Figure 1: (a) Director deformation in the splay-bend mode. Left-side figure shows three 22 dimensional image of director configuration. Ellipsoids of right-side figure exhibit the director

23 configuration at certain planes perpendicular to helical axis, and splay (top and bottom planes) and 24 bend (middle plane) deformation are observed. (b) Director deformation in the undulation mode. 25 Ellipsoids show director configuration, and solid lines indicate the deformation of pseudo-layer 
structures from the equilibrium configuration shown in broken lines.

2

3 Figure 2: (a) Splay-bend and (b) undulation mode scattering geometries. $\mathbf{q}$ is the scattering vector 4 and $\mathbf{q}_{0}$ is the wave number vector of the cholesteric helical pitch.

6 Figure 3: Autocorrelation functions measured in the two scattering geometries. Open circle and 7 square show the data measured in the splay-bend and undulation geometries respectively, and well 8 fitted by double and single exponential curves respectively. The scattering angle is $24^{\circ}$, the 9 temperature $30^{\circ} \mathrm{C}$, and the physical pitch length of the sample $430 \mathrm{~nm}$.

11 Figure 4: Dispersion relations of the two modes, (a) splay-bend and (b) undulation modes in various 12 mixtures. Fitted straight lines calculated by eq. (8a) and (8b) respectively are also shown, and it is 13 found that the slope of the fitted lines is independent of the helical pitch. Fitting parameters are $\left(K_{1}\right.$ $\left.14+K_{3}\right) / 2 \gamma_{1}=(1.1 \pm 0.2) \times 10^{-10} \mathrm{~m}^{2} / \mathrm{s}$ and $3 K_{3} / 8 \eta=(1.8 \pm 0.2) \times 10^{-11} \mathrm{~m}^{2} / \mathrm{s}$.

15

16 Figure 5: Extrapolation values of relaxation frequency of the splay-bend mode in the limit $q \rightarrow 0$ as a

17 function of square of wave number of the helical pitch. Broken line is fitted using eq. (8a), in 18 which $4 K_{2} / \gamma_{1}$ is $7.2 \times 10^{-11} \mathrm{~m}^{2} / \mathrm{s}$. 


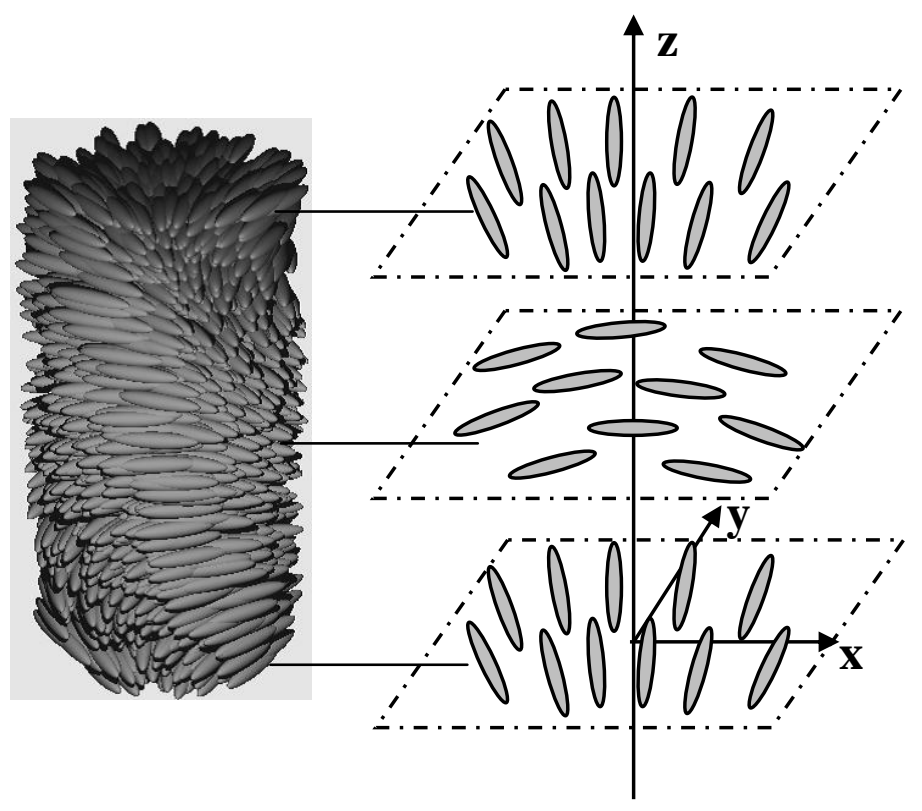

(a)

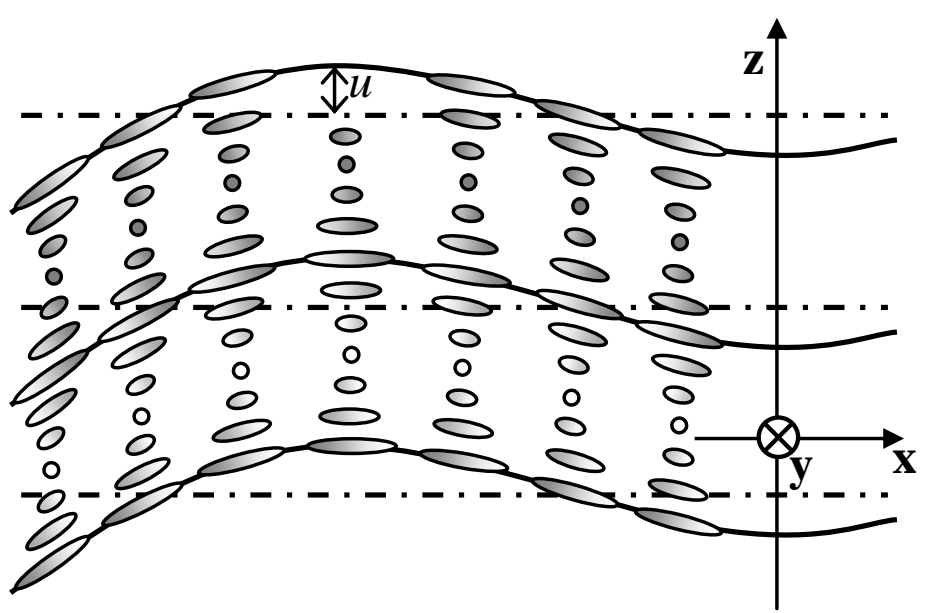

(b)

Fig.1 

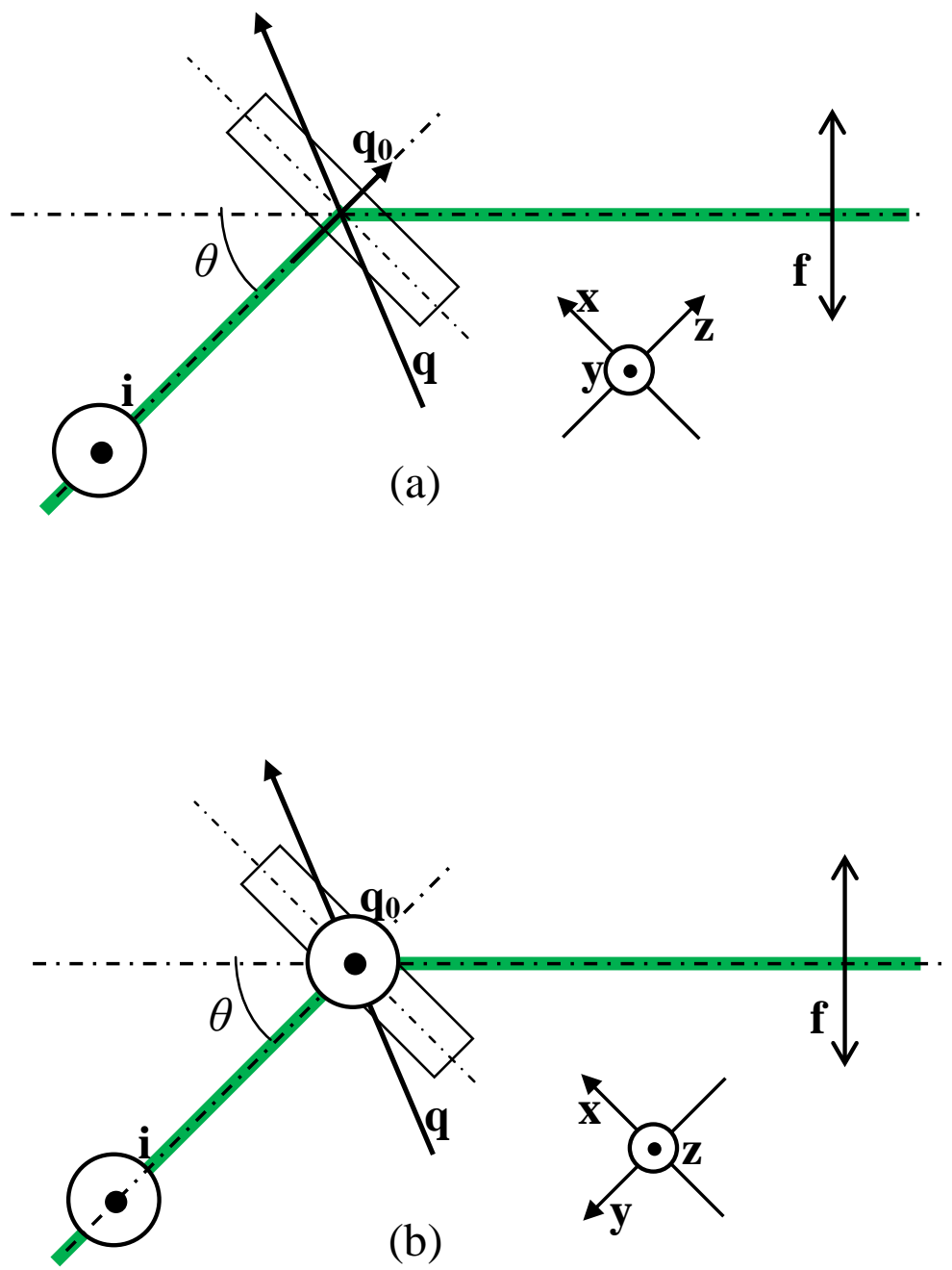

Fig. 2 
Figure 3

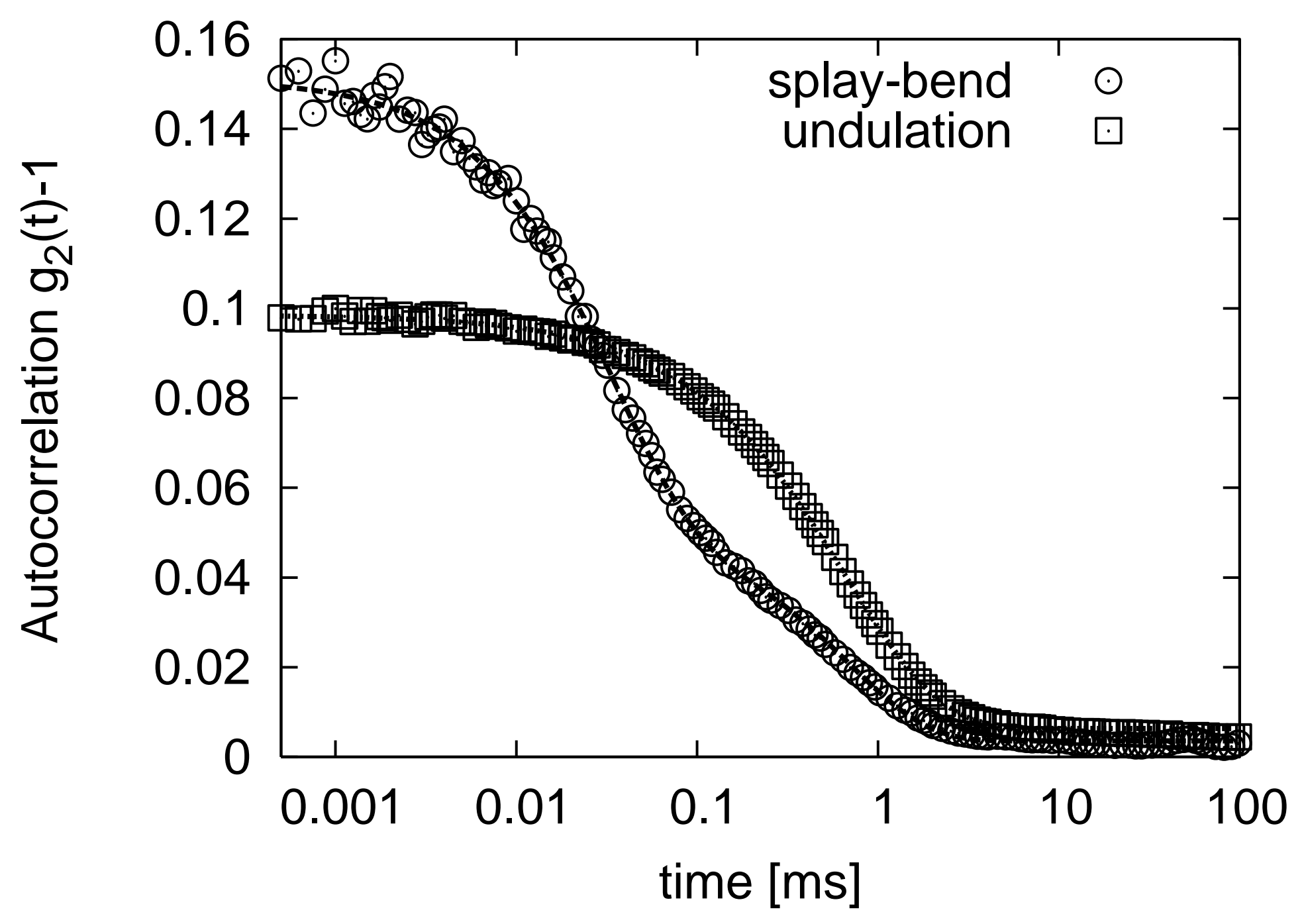


Figure 4(a)

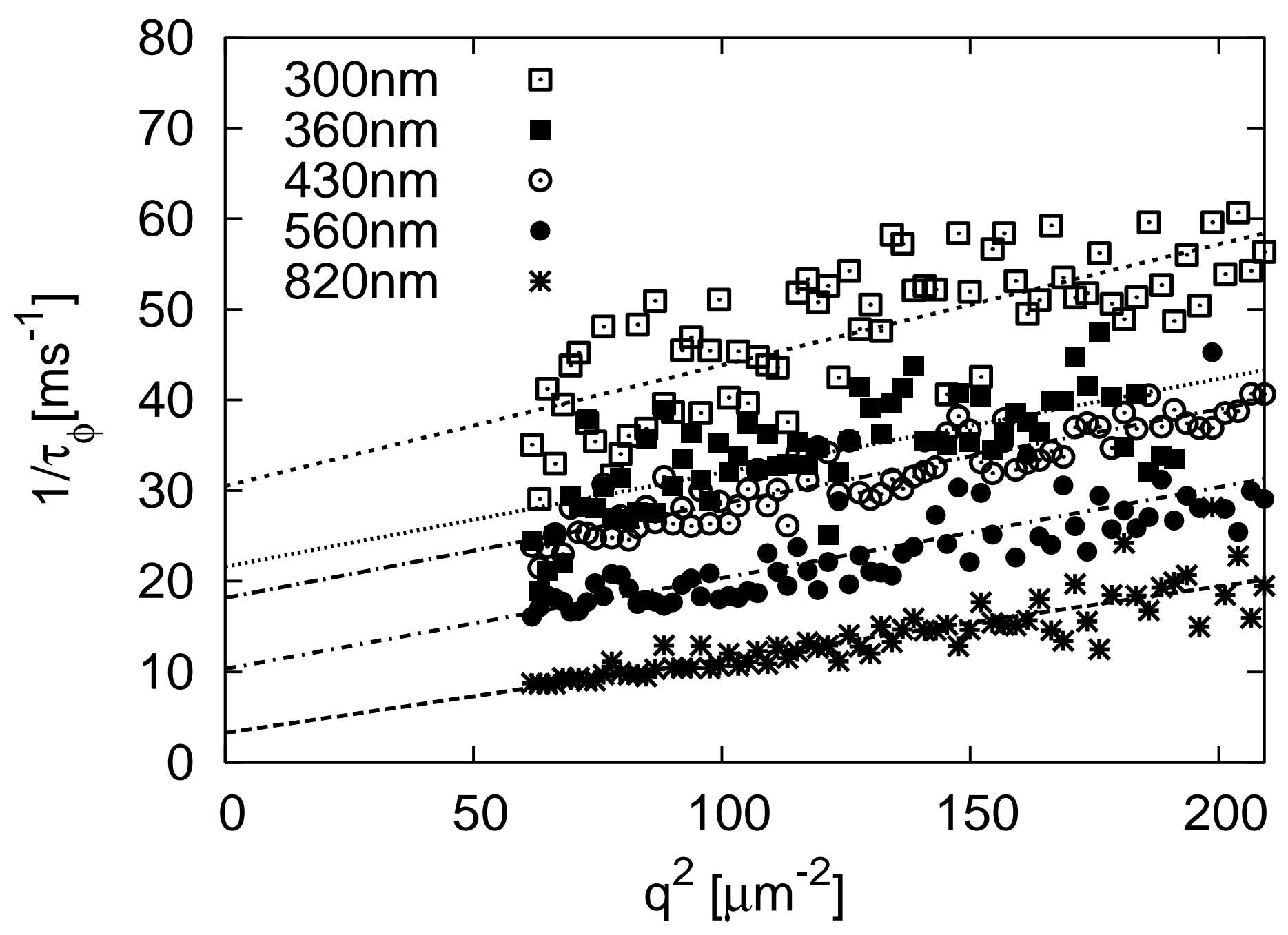


Figure 4(b)

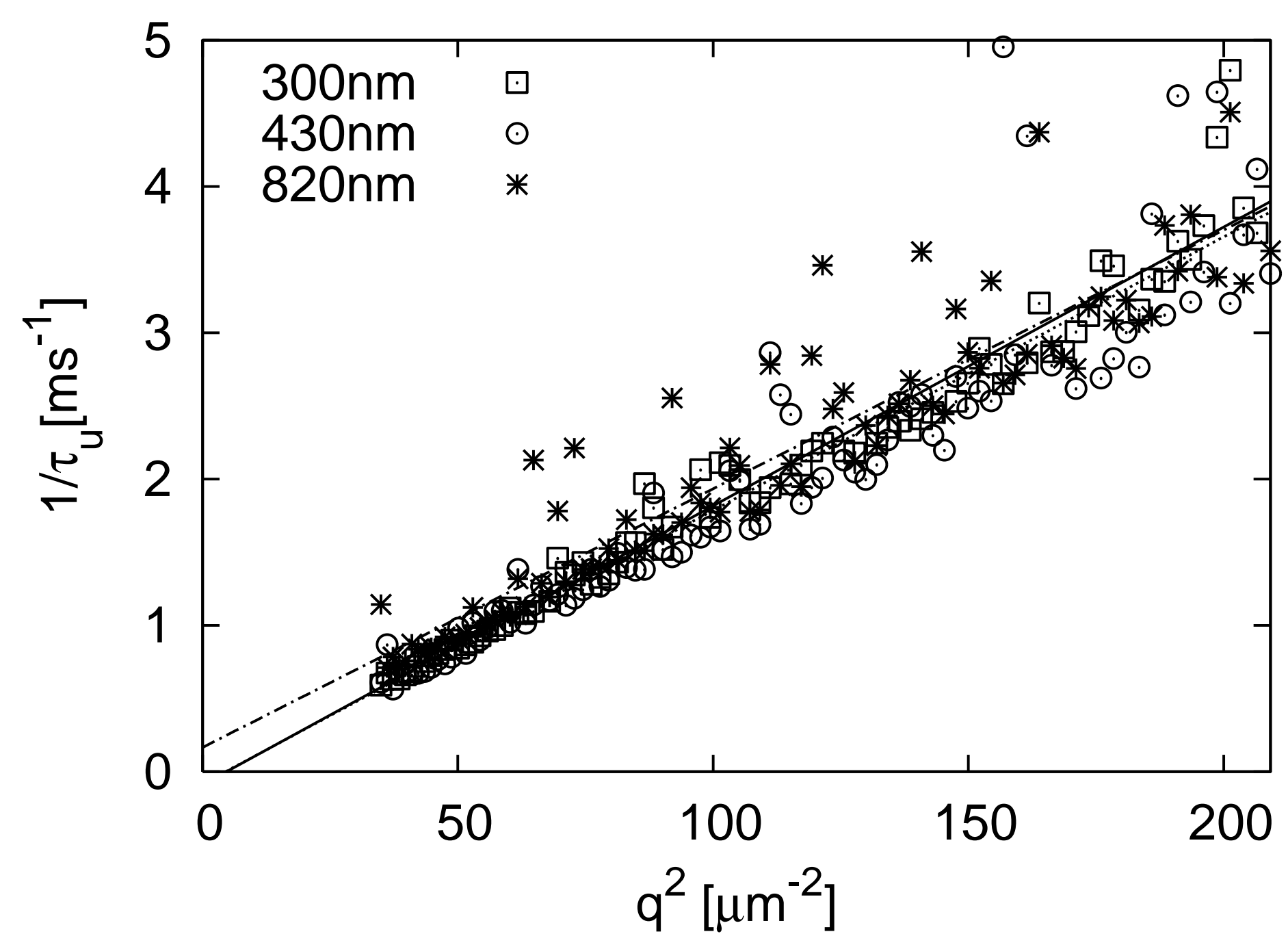


Figure 5

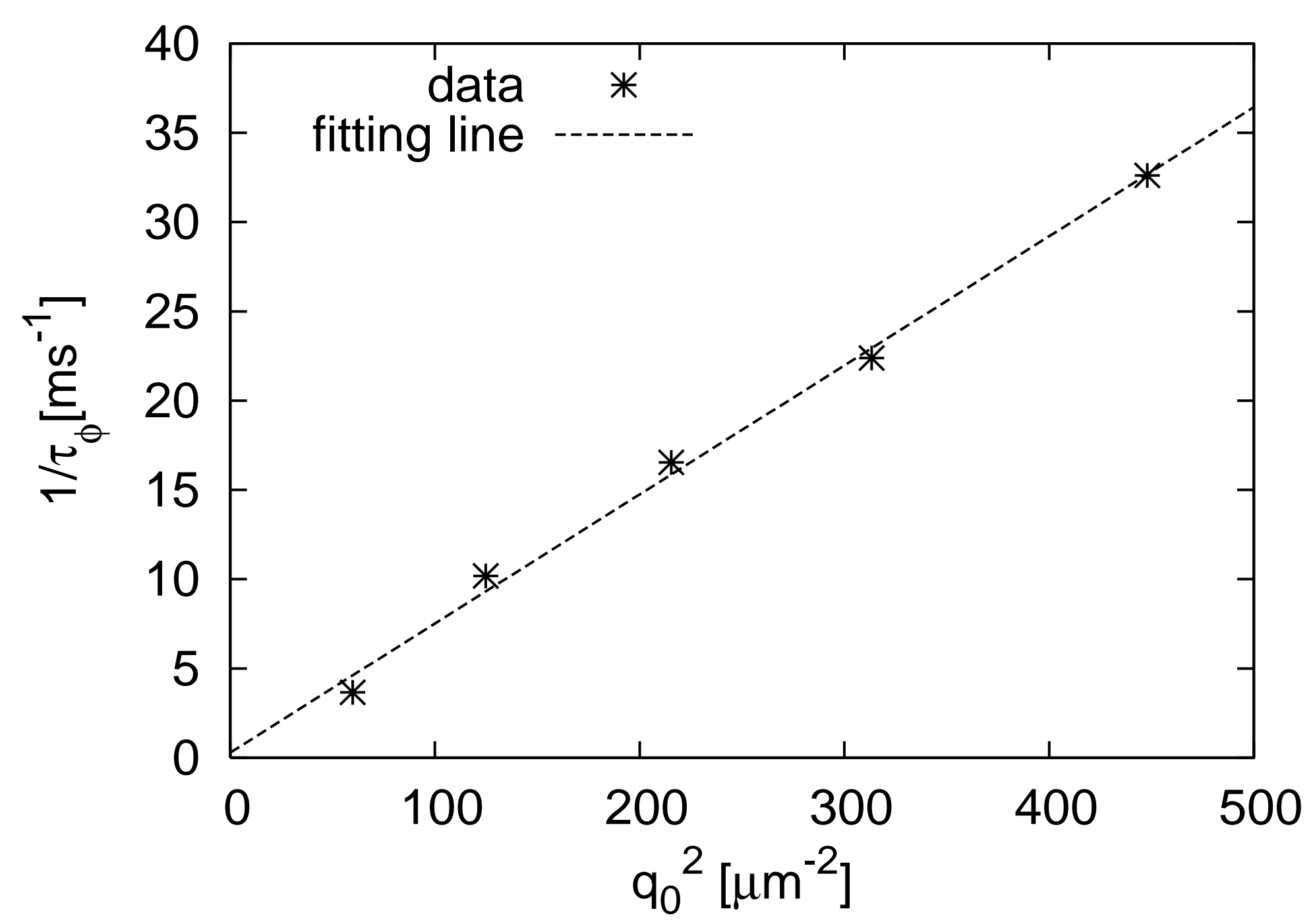

